\title{
Computing the weights of criteria with interval-valued fuzzy sets for MCDM problems
}

Chen-Tung Chen ${ }^{1}$, Kuan-Hung Lin $^{2}$, Hui-Ling Cheng ${ }^{3}$

\author{
${ }^{1}$ Department of Information Management, National United University, Taiwan \\ ${ }^{2}$ Specialist 2, Quanta Computer Inc., 211, Wen Hwa 2nd Road, Kueishan, Taoyuan, Taiwan \\ ${ }^{3}$ College of General Education, Hung Kuang University, Taiwan
}

\begin{abstract}
In the real world, many influenced factors or criteria should be considered in the multiple criteria decisionmaking (MCDM) process. Face to the uncertain environment, the decision makers or experts always cannot express their opinions exactly in the decision-making process. Under this situation, interval-valued fuzzy sets are suitable used to represent the subjective judgments of decision makers. In this paper, the fuzzy AHP method is applied to compute the fuzzy weights of each criterion based on the interval-valued fuzzy sets. The proposed method can provide a more flexible way for decision makers to express their subjective opinions. A systematic method is presented here to compute the fuzzy weights of criteria for dealing with a MCDM problem. An example is presented to illustrate the computational procedure of the proposed method. Conclusions and future research direction will be discussed at the end of this paper.
\end{abstract}

Keywords-MCDM, Interval-valued fuzzy set, Fuzzy AHP.

\section{INTRODUCTION}

Decision making is one of the most complex administrative processes in management area (Ashtiani et al., 2009). In some situations, because of time pressure, lack of knowledge, and the decision maker's limited attention and information processing capabilities (Xu, 2006), the decision maker cannot make the optimal decision easily. In fact, both qualitative and quantitative criteria should be considered simultaneously (Erol and Ferrell Jr, 2003) in the decision making process. It calls multiple criteria decision making (MCDM) process. Saaty (1980) proposed analytic hierarchy process (AHP) method for determining the weights of criteria in a MCDM problem by a hierarchical structure and systematization. According to the pairwise comparison result, the weight of each criterion can be computed for a hierarchical problem structure.

Due to the subjective judgments of the decision makers are often uncertain in determining the pairwise comparisons, the fuzzy AHP are proposed to overcome the drawback (Buckley, 1985; Buckley et al., 2001). The fuzzy AHP allowed the experts to use fuzzy ratios in place of exact ratios to make the pairwise comparisons. Based on the concepts, there are various fuzzy AHP methods have been developed such as the geometric mean method (Buckley, 1985), the Lambda-Max method (Csutora and Buckley, 2001), fuzzy logarithmic least squares method (Wang et al., 2006), the linear goal programming method (Jung, 2011), and the logarithmic fuzzy preference programming method (Rezaei et al., 2013).

However, it is often difficult for experts to exactly quantify their opinions as a crisp number in decision making process. Therefore, the reasonable way is to represent the degree of certainty by an interval (Ashtiani et al., 2009; Vahdani et al., 2010). The membership value express as an interval value can more fit the real world situations. Interval-valued fuzzy numbers have been applied in many fields such as R\&D leader selection (Ashtiani et al., 2009), risk analysis (Wei and Chen, 2009), enterprise partner selection (Ye, 2010), traditional supplier selection (Chen, 2011), bus enterprise performance evaluation (Kuo and Liang, 2012) etc. Therefore, interval-valued fuzzy sets are suitable used to represent the fuzziness of membership value of subjective opinions. In this paper, a novel method is proposed by combining interval-valued fuzzy set with fuzzy AHP to determine the fuzzy weights of each criterion.

The organization of this paper is described as follows. In section 2, we will present some notations of basic definitions and operators such as triangular fuzzy number, $\alpha$-cut of fuzzy number, defuzzified method and interval valued fuzzy sets. The proposed method will be discussed at the section 3. For illustrating the proposed method clearly, an example will be implemented. Finally, the conclusion and future research are summarized at the end of this paper.

\section{FUZZY NUMBERS AND LINGUISTIC VARIABLES}

\subsection{Triangular fuzzy number}

Triangular fuzzy number (TFN) can be defined as $\tilde{T}=(l, m, u)$, where $l<m<u$. When $l>0$, then $\tilde{T}$ is a positive TFN 
(PTFN) (Zimmerman, 1991; Chen, 2000). The membership function of positive TFN $\tilde{T}$ can be defined as follow.

$$
\mu_{\tilde{T}}(x)=\left\{\begin{array}{cc}
\frac{x-l}{m-l}, & l \leq x \leq m \\
\frac{u-x}{u-m}, & m \leq x \leq u \\
0, & \text { otherwis }
\end{array}\right.
$$

The $\alpha$-cut is used to transform a fuzzy set into crisp interval (Zimmerman, 1991). The $\alpha$-cut can define as follow.

$$
\tilde{T}^{\alpha}=[(m-l) \alpha+l, u-(u-m) \alpha], 0 \leq \alpha \leq 1
$$

In a short, it can be represented as $\tilde{T}^{\alpha}=\left[T_{l}^{\alpha}, T_{u}^{\alpha}\right]$. If $\tilde{T}$ is a triangular fuzzy number, the area measurement method can be defined as (Yager, 1981).

$$
I(\tilde{T})=\left(I_{L}(\tilde{T})+I_{R}(\tilde{T})\right) / 2
$$

where the $I_{L}(\tilde{T})$ represents the area bounded by the left-shape function of $\tilde{T}$, the area include the $\mathrm{x}$-axis, the y-axis, the horizontal line $\alpha=1$ and the line $L_{\tilde{T}}(x)$. The $I_{R}(\tilde{T})$ represents the area bounded by the right-shape function, the area include the $\mathrm{x}$-axis, the $\mathrm{y}$-axis, the horizontal line by $\alpha=1$ and the line $R_{\widetilde{T}}(x)$.

\subsection{Interval-valued fuzzy set}

The definition of interval-valued fuzzy set can be described as follow (Ashtiani et al., 2009; Chen, 2012).

$$
\begin{aligned}
& \tilde{T}=\left\{\left(x,\left[\mu_{\tilde{T}}^{L}(x), \mu_{\widetilde{T}}^{U}(x)\right]\right)\right\} \\
& \mu_{T}^{L}, \mu_{T}^{U}: X \rightarrow[0,1], \forall x \in X, \mu_{T}^{L}<\mu_{T}^{U}
\end{aligned}
$$

Yao and Lin (2002) presented the interval-valued triangular fuzzy number $\tilde{T}=\left[\mu_{\tilde{T}}^{L}, \mu_{\tilde{T}}^{U}\right]=[(l l, m, u ; \rho),(l, m, u u ; \lambda)](\operatorname{shown}$ as Fig. 1), where the $\mu_{T}^{L}$ denotes the lower limit degree of membership and the $\mu_{T}^{U}$ denotes the upper limit degree of membership, and the $\mu_{T}^{L} \subset \mu_{T}^{U}$.

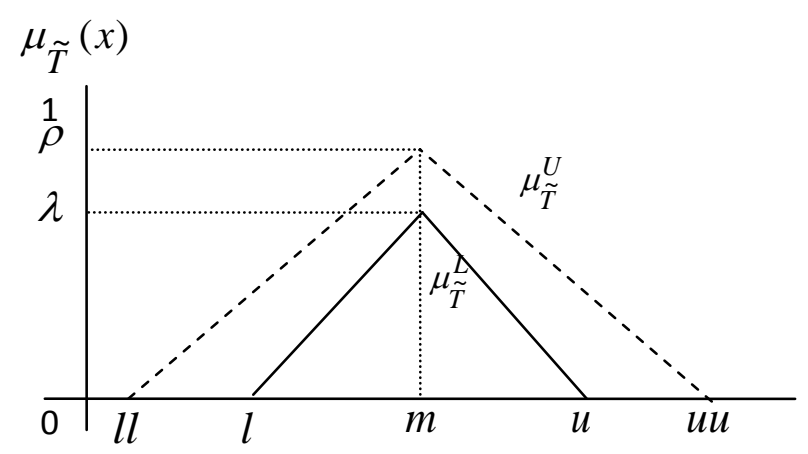

\section{FIG. 1. INTERVAL-VALUED TRIANGULAR FUZZY NUMBER}

In order to express the fuzziness more exactly, Ashtiani et. al., (2009) and Vahdani et. al (2010) used triangular intervalvalued fuzzy numbers to represent the opinions of decision-makers. In many cases, an expert cannot exactly express the opinion by a crisp membership value. Under this situation, the interval-valued fuzzy number can express the uncertain opinion as an interval value. The membership value of $x$ can be extended as $\left[\mu_{\widetilde{T}}^{L}(x), \mu_{\tilde{T}}^{U}(x)\right]$. In this paper, we suppose that the lower limit degree of membership and upper limit degree of membership at $m$ are equal to 1 . It represents a normal interval-valued triangular fuzzy number (IFVNs). 
Assume that there are two interval-valued triangular fuzzy numbers $\tilde{T}_{1}$ and $\tilde{T}_{2}$, where $\tilde{T}_{1}=\left(\left[l l_{1}, l_{1}\right], m_{1},\left[u_{1}, u u_{1}\right]\right.$ and $\tilde{T}_{2}=\left(\left[l l_{2}, l_{2}\right], m_{2},\left[u_{2}, u u_{2}\right]\right.$, the arithmetic operations can be calculated as follows (Kuo and Liang, 2012).

$$
\begin{aligned}
& \tilde{T}_{1}+\tilde{T}_{2}=\left(\left[l l_{1}+l l_{2}, l_{1}+l_{2}\right], m_{1}+m_{2},\left[u_{1}+u_{2}, u u_{1}+u u_{2}\right]\right) \\
& \tilde{T}_{1}-\tilde{T}_{2}=\left(\left[l l_{1}-u u_{2}, l_{1}-u_{2}\right], m_{1}-m_{2},\left[u_{1}-l_{2}, u u_{1}+l l_{2}\right]\right) \\
& \tilde{T}_{1} \otimes \tilde{T}_{2} \approx\left(\left[l l_{1} \otimes l l_{2}, l_{1} \otimes l_{2}\right], m_{1} \otimes m_{2},\left[u_{1} \otimes u_{2}, u u_{1} \otimes u u_{2}\right]\right) \\
& \tilde{T}_{1} \div \tilde{T}_{2} \approx\left(\left[l l_{1} \div u u_{2}, l_{1} \div u_{2}\right], m_{1} \div m_{2},\left[u_{1} \div l_{2}, u u_{1} \div l l_{2}\right]\right)
\end{aligned}
$$

\section{PROPOSED METHOD}

Based on interval-valued fuzzy sets, the Lambda-Max method (Csutora and Buckley, 2001) is applied to compute the fuzzy weight of each criterion. The computational procedure is shown as follows.

\section{Step 1. Establish hierarchical structure.}

Establish a hierarchical framework of decision-making problem. Within the hierarchical framework, there may contain several criterion and sub-criterion.

Step 2. The expert uses the interval-valued linguistic variable to make the pairwise comparison in the criteria and sub-criteria levels.

Step 3. Using interval-valued fuzzy AHP, the weights of criteria and sub-criteria can be computed and expressed as intervalvalued fuzzy numbers. The computational procedure of calculation steps as follow.

\section{1) Construct the fuzzy positive reciprocal matrix.}

The experts are allowed to use interval-valued fuzzy ratios (see Table 1 ) in place of exact ratios. The $\tilde{T}_{i j}, \mathrm{i} \neq \mathrm{j}$, can now be interval-valued fuzzy numbers in any positive reciprocal matrix. The fuzzy positive reciprocal matrix is defined as $\tilde{T}=\left[\tilde{T}_{i j}\right]_{n \times n}, \quad$ where $\quad \tilde{T}_{i j}=1, \forall i=j, \quad \tilde{T}_{j i}=\frac{1}{\tilde{T}_{i j}}, \forall i, j=1,2, \ldots, n$.If $\quad \tilde{T}_{i j}=([l l, l] m,[u, u u]), \quad$ then $\tilde{T}_{i j}^{-1}=\left(\left[u u^{-1}, u^{-1}\right], m^{-1},\left[l^{-1}, l l^{-1}\right]\right)$.

\section{TABLE 1}

LINGUISTIC VARIABLE FOR IMPORTANCE

\begin{tabular}{|c|c|c|}
\hline Symbol & Linguistic variables & Interval-value fuzzy number \\
\hline (1) & Extremely Equal Importance & $([1,1], 1,[1,1])$ \\
\hline (2) & Equal Importance & $([1,1], 1,[2,3]), 2,[3,4])$ \\
\hline (3) & Intermediate values & $([1,2], 3,[4,5])$ \\
\hline (4) & Weak Importance & $([2,3], 4,[5,6])$ \\
\hline (5) & Intermediate values & $([3,4], 5,[6,7])$ \\
\hline (6) & Essential Importance & $([4,5], 6,[7,8])$ \\
\hline (7) & Intermediate values & $([5,6], 7,[8,9])$ \\
\hline (8) & Very Strong Importance & $([6,7], 8,[9,9])$ \\
\hline (9) & Intermediate values & $([7,8], 9,[9,9])$ \\
\hline (10 & Absolute Importance & $(5,1)$ \\
\hline
\end{tabular}




\section{2) Compute the interval-valued fuzzy weights of criteria.}

According to the Lambda-Max method (Csutora and Buckley, 2001), a sequence of fuzzy positive reciprocal matrix is applied to determine the relative weights of each criterion. The procedure of all calculation steps as follow.

a. Let $\alpha=1$, using $\alpha$-cut to obtain $\tilde{T}_{m}^{1}=\left[t_{i j m}\right]_{n \times n}$, it represent the decision-makers give the crisp positive reciprocal matrix $\tilde{T}$, and determine the fuzzy weight $\tilde{W}_{m}$, for $\tilde{W}_{m}=\left[W_{i m}\right], i=1,2, \ldots, n$.

b. Let $\alpha=0$, using $\alpha$-cut to obtain the $\tilde{T}_{u}^{0}=\left[t_{i j u}\right]_{n \times n}, \quad \tilde{T}_{u u}^{0}=\left[t_{i j u u}\right]_{n \times n}, \quad \tilde{T}_{l l}^{0}=\left[t_{i j l l}\right]_{n \times n}$, and $T_{l}^{0}=\left[t_{i j l}\right]_{n \times n}$ in accordance with the fuzzy positive reciprocal matrix. Next, computing the fuzzy weights $\tilde{W}_{l l}, \tilde{W}_{l}, \tilde{W}_{u}$ and $\tilde{W}_{u u}$, where $\tilde{W}_{l l}=\left[W_{i l l}\right], \tilde{W}_{l}=\left[W_{i l}\right], \tilde{W}_{u}=\left[W_{i u}\right]$, and $\tilde{W}_{u u}=\left[W_{i u u}\right], i=1,2, \ldots, n$.

c. In order to make sure the fuzzy weights are still normal fuzzy sets, the following formula is used to adjust these weights. First, we can determine lower bounds triangular fuzzy weights as $Q_{l}=\min \left\{\frac{W_{i m}}{W_{i l}} \mid 1 \leq i \leq n\right\}$ and $Q_{u}=\max \left\{\frac{W_{i m}}{W_{i u}} \mid 1 \leq i \leq n\right\}$

The constants $Q_{l}$ and $Q_{u}$ are used to calculate the new weight as $W_{i l}^{*}=Q_{l} \times W_{i l}$ and $W_{i u}^{*}=Q_{u} \times W_{i u}$.

Second, we use the new fuzzy weight of lower limit and upper limit to determine the upper bounds triangular fuzzy weights as $Q_{l l}=\min \left\{\frac{W_{i l}^{*}}{W_{i l l}} \mid 1 \leq i \leq n\right\}$ and $Q_{u u}=\max \left\{\frac{W_{i u}^{*}}{W_{i u u}} \mid 1 \leq i \leq n\right\}$.

Then, the constants $Q_{l l}$ and $Q_{u u}$ are used to determine the new upper bounds triangular fuzzy weights as $W_{i l l}^{*}=Q_{l l} \times W_{i l l}$ and $W_{i u u}^{*}=Q_{u u} \times W_{i u u}$.

Finally, we can get the new fuzzy weights $W_{l l}^{*}=\left[W_{i l l}^{*}\right], W_{l}^{*}=\left[W_{i l}^{*}\right], W_{u}^{*}=\left[W_{i u}^{*}\right]$, and $W_{u u}^{*}=\left[W_{i u u}^{*}\right]$, for $\mathrm{i}=1,2, \ldots \mathrm{n}$.

d. To integrate $W_{l l}^{*}, W_{l}^{*}, W_{m}, W_{u}^{*}$ and $W_{u u}^{*}$, we can obtain the positive triangular fuzzy weight matrix for decisionmakers. The fuzzy weights of $i$-th criterion can be represented as $\tilde{W}_{i}=\left(\left[W_{i l l}^{*}, W_{i l}^{*}\right], W_{i m},\left[W_{i u}^{*}, W_{i u u}^{*}\right]\right)$.

e. Defuzzy the interval-valued fuzzy weights.

Applying the area measurement method (Yager, 1981) to transfer the interval-valued fuzzy number into interval value as $W_{j}=\left[W_{j}^{\min }, W_{j}^{\max }\right]$, where $W_{j}$ represents the interval weight of criterion $j$, the $W_{j}^{\min }=\frac{W_{j l l}+2 W_{j m}+W_{j l}}{4}$ represents the lower limit of the interval and $W_{j}^{\max }=\frac{W_{j u}+2 W_{j m}+W_{j u u}}{4}$ represents the upper limit of the interval.

3) Repeating the process of step (B), the weights of sub-criteria can be computed with respect to each criterion.

\section{NUMERICAL EXAMPLE}

Suppose that a company desires to select an information system to serve their customer. A committee of three decisionmakers (or experts) $\left(p_{1}, p_{2}, p_{3}\right)$ has been formed to evaluate the importance of each criterion. After the reviewing by the committee, three criteria and nine sub-criteria $\left(C_{11}, C_{12}, \ldots, C_{33}\right)$ are considered to select the suitable vendor. The computation process of proposed method is shown as follows.

Step 1. According to the description, the hierarchical structure of problem is shown as Fig.2.

Step 2. Each expert uses the interval-value linguistic variables to make the pairwise comparisons among criteria as Table 2. 


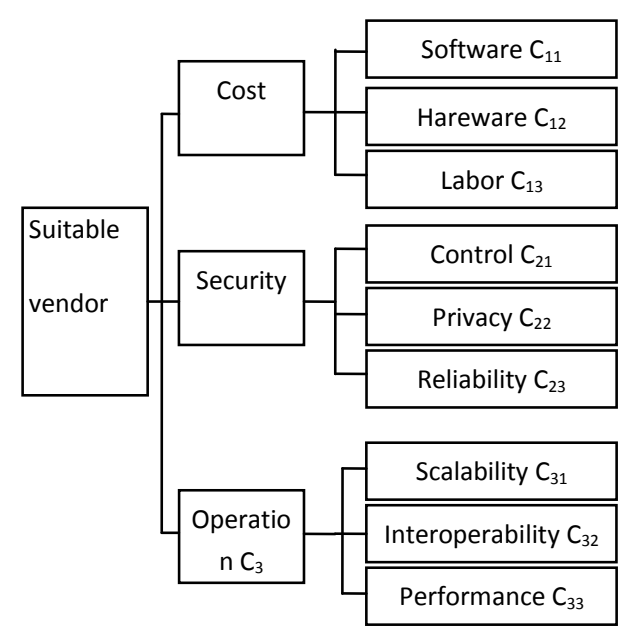

Fig. 2. THE EVALUATION STRUCTURE

TABLE 2

PAIRWISE COMPARISONS OF THREE EXPERTS

\begin{tabular}{|c|c|c|c|c|c|c|c|c|c|c|c|c|c|c|c|c|}
\hline \multirow{4}{*}{$P_{1}$} & & $C_{1}$ & $C_{2}$ & $C_{3}$ & & $C_{11}$ & $C_{12}$ & $C_{13}$ & & $C_{21}$ & $C_{22}$ & $C_{23}$ & & $C_{31}$ & $C_{32}$ & $C_{33}$ \\
\hline & $C_{1}$ & (1) & (3) & (5) & $C_{11}$ & (1) & $(6)^{-1}$ & $(8)^{-1}$ & $C_{21}$ & (1) & (4) & (6) $)^{-1}$ & $C_{31}$ & (1) & $\left(5^{-1}\right.$ & (3) \\
\hline & $C_{2}$ & $\left(^{(3)^{-1}}\right.$ & (1) & $(2)^{-1}$ & $C_{12}$ & (6) & (1) & $\left(^{(3)}\right)^{-1}$ & $C_{22}$ & $\left(4^{-1}\right.$ & (1) & $\left(\right.$ (10) $^{-1}$ & $C_{32}$ & (5) & (1) & (9) \\
\hline & $C_{3}$ & $(5)^{-1}$ & (2) & (1) & $C_{13}$ & (8) & (3) & (1) & $C_{23}$ & (6) & (10) & (1) & $C_{33}$ & $(3)^{-1}$ & $(9)^{-1}$ & (1) \\
\hline \multirow{4}{*}{$P_{2}$} & & $C_{1}$ & $C_{2}$ & $C_{3}$ & & $C_{11}$ & $C_{12}$ & $C_{13}$ & & $C_{21}$ & $C_{22}$ & $C_{23}$ & & $C_{31}$ & $C_{32}$ & $C_{33}$ \\
\hline & $C_{1}$ & (1) & (6) & (5) & $C_{11}$ & (1) & $(3)^{-1}$ & (2) -1 & $C_{21}$ & (1) & $(7)^{-1}$ & (4) & $C_{31}$ & (1) & (5) & (3) \\
\hline & $C_{2}$ & $(6)^{-1}$ & (1) & (3) & $C_{12}$ & (3) & (1) & (5) -1 & $C_{22}$ & (7) & (1) & (8) & $C_{32}$ & $(5)^{-1}$ & (1) & (8) \\
\hline & $C_{3}$ & $(5)^{-1}$ & $(3)^{-1}$ & (1) & $C_{13}$ & (2) & (5) & (1) & $C_{23}$ & $(4)^{-1}$ & $(8)^{-1}$ & (1) & $C_{33}$ & (3) ${ }^{-1}$ & $(8)^{-1}$ & (1) \\
\hline \multirow{4}{*}{$P_{3}$} & & $C_{1}$ & $C_{2}$ & $C_{3}$ & & $C_{11}$ & $C_{12}$ & $C_{13}$ & & $C_{21}$ & $C_{22}$ & $C_{23}$ & & $C_{31}$ & $C_{32}$ & $C_{33}$ \\
\hline & $C_{1}$ & (1) & $(7)^{-1}$ & $(6)^{-1}$ & $C_{11}$ & (1) & (4) & (3) & $C_{21}$ & (1) & (5) & (9) & $C_{31}$ & (1) & $(3)^{-1}$ & $\left(9^{-1}\right.$ \\
\hline & $C_{2}$ & (7) & (1) & $(3)^{-1}$ & $C_{12}$ & $(4)^{-1}$ & (1) & (5) & $C_{22}$ & $(5)^{-1}$ & (1) & (3) & $C_{32}$ & (3) & (1) & $\left(5^{-1}\right.$ \\
\hline & $C_{3}$ & (6) & (3) & (1) & $C_{13}$ & $\left(^{(3)}\right)^{-1}$ & $\left(5^{-1}\right.$ & (1) & $C_{23}$ & $(9)^{-1}$ & $\left(^{(3)}\right)^{-1}$ & (1) & $C_{33}$ & (9) & (5) & (1) \\
\hline
\end{tabular}

Step 3. Compute the interval-valued fuzzy weights of criteria.

i. The fuzzy positive reciprocal matrix is shown as Table 2 .

ii. According to the fuzzy reciprocal matrix for three criteria, the criteria weights of the expert $P_{1}$ can be computed as follows

(1) Let $\alpha=1$, using $\alpha$-cut to obtain $\widetilde{T}_{m}^{1}=\left[\begin{array}{ccc}1 & 2 & 4 \\ 1 / 2 & 1 & 1 \\ 1 / 4 & 1 & 1\end{array}\right]$, and determine the fuzzy weight as $W_{m}=[0.584,0.232,0.184]$.

(2) Let $\alpha=0$, using $\alpha$-cut to obtain the positive reciprocal matrix as $\tilde{T}_{l l}^{0}=\left[\begin{array}{ccc}1 & 1 & 2 \\ 1 / 4 & 1 & 1 / 3 \\ 1 / 6 & 1 & 1\end{array}\right], \quad \tilde{T}_{l}^{0}=\left[\begin{array}{ccc}1 & 1 & 3 \\ 1 / 3 & 1 & 1 / 2 \\ 1 / 5 & 1 & 1\end{array}\right]$, $\tilde{T}_{u}^{0}=\left[\begin{array}{ccc}1 & 3 & 5 \\ 1 & 1 & 1 \\ 1 / 3 & 2 & 1\end{array}\right], \quad \tilde{T}_{u u}^{0}=\left[\begin{array}{ccc}1 & 4 & 6 \\ 1 & 1 & 1 \\ 1 / 2 & 3 & 1\end{array}\right]$. Next, we can compute the fuzzy weights of each matrix as $W_{l l}^{0}=[0.570,0.189,0.241], W_{l}^{0}=[0.579,0.204,0.217], W_{u}^{0}=[0.553,0.239,0.208]$,

$W_{u u}^{0}=[0.565,0.205,0.230]$.

(3) In order to make sure the weights are still normal fuzzy sets, first we adjust lower bounds triangular fuzzy weights and find the constants $Q_{l}=0.849$ and $Q_{u}=1.056$. And then, obtain the new weights $W_{l}^{*}=[0.491,0.173,0.184]$ and 
$W_{u}^{*}=[0.584,0.253,0.220]$. Next we adjust upper bounds triangular fuzzy weights, and find the constants $Q_{l l}=0.763$ and $Q_{u u}=1.236$. Then, obtain the new weights $W_{l l}^{*}=[0.435,0.144,0.184]$ and $W_{u u}^{*}=[0.699,0.253,0.285]$.

(4) To combine the weight matrix $\left[W_{l l}^{*}, W_{l}^{*}\right], W_{m},\left[W_{u}^{*}, W_{u u}^{*}\right]$, we can obtain the interval-valued fuzzy weight of expert $P_{1}$ for three criteria as

$$
\begin{gathered}
\tilde{W}_{1}=([0.435,0.491], 0.584,[0.584,0.699]) \tilde{W}_{2}=([0.144,0.173], 0.232,[0.253,0.253]) \\
\tilde{W}_{3}=([0.184,0.184], 0.184,[0.220,0.285])
\end{gathered}
$$

(5) Integrate the interval-valued fuzzy weights of three experts; the area measurement method is applied to transform the interval-valued fuzzy set into interval value. The weights of criteria can be computed as $W_{1}=[0.422,0.454], W$ ${ }_{2}=[0.256,0.264]$ and $W_{3}=[0.287,0.303]$.

iii. The computational procedures of sub-criteria weights are illustrated as step B and the result can be shown as Table 3 .

TABLE 3

THE WEIGHTS OF SUB-CRITERIA

\begin{tabular}{|c|c|c|c|c|c|}
\hline Sub-criteria & Weights & Sub-criteria & Weights & Sub-criteria & Weights \\
\hline$C_{11}$ & {$[0.261,0.283]$} & $C_{21}$ & {$[0.347,0.366]$} & $C_{31}$ & {$[0.267,0.285]$} \\
\hline$C_{12}$ & {$[0.299,0.316]$} & $C_{22}$ & {$[0.259,0.341]$} & $C_{32}$ & {$[0.409,0.424]$} \\
\hline$C_{13}$ & {$[0.416,0.439]$} & $C_{23}$ & {$[0.289,0.307]$} & $C_{33}$ & {$[0.308,0.317]$} \\
\hline
\end{tabular}

\section{CONCLUSIONS AND FUTURE RESEARCH}

In general, many quantitative and qualitative factors will influence the expert to make a decision in the decision-making process. Under this situation, decision makers always difficult to express their opinions by the membership value exactly. Therefore, the interval-valued fuzzy sets are suitable to express the subjective opinion of each decision maker in a decision environment. In this paper, the fuzzy AHP method is extended to compute the fuzzy weights of all criteria based on the interval-value fuzzy sets. Applying the proposed method, a systematic way is presented here to compute the fuzzy weights of criteria. In the future, a decision analysis system will be designed and developed based on this proposed method to reduce the computational time and improve the decision-making quality.

\section{ACKNOWLEDGEMENT}

This work was supported partially by Ministry of Science and Technology, Taiwan. The project No. is "MOST 105-2410-H239-006-MY2".

\section{REFERENCES}

[1] B. Ashtiani, F. Haghighirad, A. Makui and G. A. Montazer, "Extension of fuzzy TOPSIS method based on interval-valued fuzzy sets," Applied Soft Computing, Vol.9, 2009, pp.457-461.

[2] J. J. Buckley, "Fuzzy hierarchical analysis," Fuzzy Sets and Systems, Vol.17, 1985, pp.233-247.

[3] J. J.Buckley, T. Feuring, and Y. Hayashi, "Fuzzy hierarchical analysis revisited," Fuzzy Sets and Systems, Vol.129, 2001, pp. 48-64.

[4] C. T. Chen, "Extensions of the TOPSIS for group decision-making under fuzzy environment," Fuzzy sets and systems, Vol.114, No. 1, 2000, pp.1-9.

[5] T. Y. Chen, "Optimistic and pessimistic decision making with dissonance reduction using interval-valued fuzzy sets," Information Sciences, Vol.181, No.3, 2011, pp.479-502.

[6] T. Y. Chen, "Comparative analysis of SAW and TOPSIS based on interval-valued fuzzy sets: Discussions on score functions and weight constraints," Expert Systems with Applications, Vol.39, No.2, 2012, pp.1848-1861.

[7] R. Csutora and J. J. Buckley, "Fuzzy hierarchical analysis: the Lambda-Max method," Fuzzy Sets and Systems, Vol.120, 2001, pp.181-195.

[8] I. Erol and Jr, W. G. Ferrell, "A methodology for selection problems with multiple, conflicting objectives and both qualitative and quantitative criteria," International Journal of Production Economics, Vol.86, No.3, 2003, pp.187-199.

[9] H. Jung, "A fuzzy AHP-GP approach for integrated production-planning considering manufacturing partners," Expert systems with Applications, Vol.38, No.5, 2011, pp.5833-5840. 
[10] M. S. Kuo and G. S. Liang, "A soft computing method of performance evaluation with MCDM based on interval-valued fuzzy numbers," Applied Soft Computing, Vol.12, No.1, 2012, pp.476-485.

[11] J. Rezaei, R. Ortt and V. Scholten, "An improved fuzzy preference programming to evaluate entrepreneurship orientation," Applied Soft Computing, Vol.13, No.5, 2013, pp.2749-2758.

[12] T. L. Saaty, “The Analytic Hierarchy Process,” New York: McGraw-Hill, 1980.

[13] B. Vahdani,H. Hadipour, J. S. Sadaghiani and M. Amiri,"Extension of VIKOR method based on interval-valued fuzzy sets," The International Journal of Advanced Manufacturing Technology, Vol.47, No.9-12, 2010, pp. 1231-1239.

[14] Y. M. Wang, T. Elhag and Z. Hua, "A modified fuzzy logarithmic least squares method for fuzzy analytic hierarchy process," Fuzzy Sets and Systems, Vol.157, No.23, 2006, pp.3055-3071.

[15] Z. S. Xu, “Induced uncertain linguistic OWA operators applied to group decision making,” Information Fusion, Vol.7, 2006, pp.231238.

[16] R. R. Yager, “A procedure for ordering fuzzy subsets of the unit interval,” Information Sciences, Vol.24, 1981, pp.143-161.

[17] J. S. Yao and F. T. Lin, "Constructing a fuzzy flow-shop sequencing model based on statistical data," International Journal of Approximate Reasoning, Vol.29, 2002, pp.215-234.

[18] F. Ye, "An extended TOPSIS method with interval-valued intuitionistic fuzzy numbers for virtual enterprise partner selection," Expert Systems with Applications, Vol.37, No.10, 2010, pp.7050-7055.

[19] H. J. Zimmerman, “Fuzzy Set Theory and its Applications,” Boston: Kluwer Academic Publishers, 1991. 